\title{
RELACIÓN DE LAS DROGAS CON LAS ACTITUDES SEXUALES Y VÍNCULOS .DE PAREJA. UN ESTUDIO CUALITATIVO DESCRIPTIVO-ANALÍTICO EN ADOLESCENTES MUJERES DE LIMA
}

\author{
Jorge Arnao Marciani \\ Carlos Cabezudo Moreno
}

\begin{abstract}
RESUMEN
El estudio usa una metodología cualitativa, descriptiva - analítica, tiene por finalidad interpretarlos significados del riesgo sexual de las adolescentes de Lima que acuden a espacios de diversión dentro de un contexto donde hay alcohol y drogas. La muestra está compuesta por 64 adolescentes mujeres entre los 15 y 18 años. El criterio de selección se basó en informantes clave. Se realizaron 8 grupos focales en una academia preuniversitaria del centro de Lima.

La primera embriaguez se produciría alrededor de los 15 años. Los espacios son: el término del colegio (reuniones programadas), la fiesta de promoción, los quinceañeros y los paseos de promoción. Los amigos se constituyen en el grupo social donde se dan por primera vez el ofrecimiento y/o la disponibilidad para el consumo de la marihuana.

La Pasta básica de cocaína (PBC) es la droga menos cercana a las adolescentes, estando su consumo relacionado con actitudes negativas y de rechazo definidas, a diferencia del éxtasis que se está extendiendo cada vez más hacia sectores más populares como el $C$ y $D$, apartándose rápidamente de los sectores $A$ y $B$ donde inicialmente apareció.

Coexisten dos discursos sobre la virginidad: uno el que considera que ésta debe cuidarse, y el otro que le resta valor; otorgando más relevancia a las cualidades personales en una relación. Sin embargo, las que coincidían con la primera posición manifestaban no saber que podría pasar con su virginidad en el futuro. La consecuencia de tener relaciones sexuales más temida por estas adolescentes es el embarazo, antes que el VIH.

Llama la atención que la mayoría de las adolescentes entrevistadas ha sufrido algún tipo de agresión sexual. Se encontró además que las relaciones de pareja se dan en un contexto de diversión y existirían tres tipos vínculos: los llamados "agarres", "vacilones" y enamorados.
\end{abstract}

Palabras Clave: Adolescentes, alcohol, drogas, sexualidad, vínculos de pareja, diversión.

\section{ABSTRACT}

This study uses a qualitative, descriptive analytic methodology, and interprets the significant factors of sexual risk for adolescents of Lima, who meet for recreation in a context where there exists both alcohol and drugs. The work is composed of 64 adolescent women between the ages of 15 and 18. The criterion for selection is based on unidentified informants. The focus was eight groups in a pre-university academy in the center of Lima.

The first incident of intoxication was found to be around 15 years. The situations are the following: the end of high school (planned meetings), the promotional party, the fifteen-year birthday party, and miscellaneous walks. The friends are constituted by a social group where the offer and/or the availability for the consumption of marihuana is given for the first time.

The paste base of cocaine $(P B C)$ is the drug least associated with adolescents, where its consumption is related to negative attitudes and its definite rejection, contrasted with ecstasy, which is continually growing in more popular sectors such as $C$ and $D$, and shrinking in sectors $A$ and $B$, where it had initially appeared.

There exist two points of view concerning virginity: the first affirms that it must be kept and continued, and the other places its relevance in the personal decisions of a relationship. However; those of the first position do not know if they will continue their virginity in the future. The most feared consequence of having sexual relations for these adolescents is becoming pregnant, and after that is the fear of HIV.

It should be called to attention that the majority of the adolescents interviewed have suffered some type of sexual aggression.

Furthermore, it is found that there exists three basic different types of sexual behaviour among the young in a context of recreation, known in English roughly as one, superficial physical contact, two, "hooking-up, "and three, "going-out."

Key Words: Adolescents, alcohol, drugs, sexuality, relations of a couple, recreation. 


\section{INTRODUCCIÓN}

Dado que la población adolescente es una de las más numerosas en la actualidad y se estima que en los próximos años será predominante, hemos considerado conveniente iniciar una serie de estudios de corte cualitativo que puedan ir brindándonos información más profunda sobre el mundo de los adolescentes de hoy de nuestro país. Así, este primer estudio cualitativo buscó adentrarse en los significados que otorgan las adolescentes de Lima a las drogas y el alcohol, haciendo hincapié en esta última droga porque no existen estudios que ofrezcan datos sobre ella. Precisar qué tipo de influencia y/o presencia tiene el alcohol en la sexualidad de las adolescentes fue otra interrogante que derivó al tratar de responder a la primera, ya que es inevitable preguntamos si estos episodios de consumo de drogas y/o alcohol aumentan el peligro de conductas sexuales riesgosas. Todo esto nos condujo a una interrogante más sociológica pero igualmente válida: ¿los espacios de diversión también se han adaptado a la presencia del alcohol?, ¿qué significado tiene el alcohol en estos espacios de diversión adolescente? Por ello, la presente investigación consta de tres áreas interdependientes: la primera relacionada a las drogas y el alcohol, la segunda definida por las conductas sexuales riesgosas y la tercera determinada por los espacios de diversión y las relaciones que las adolescentes establecen en ellos.

Los estudios cualitativos tienen como finalidad comprender el sentido y significado de la acción social de los sujetos dentro de la vida cotidiana. Con estos métodos se trata de ingresar al entramado de relaciones sociales compuestas por significados compartidos entre los sujetos, es decir, a la cultura de una determinada comunidad o grupo humano.

Los sujetos dan sentido a la realidad a través del lenguaje, con este se construyen las instituciones, las normas, las regularidades y las relaciones. El discurso o lenguaje nos hace comprender las acciones de los "otros", por eso a través de él accedemos al teatro de las subjetividades de los individuos, a las mentalidades, a la cultura, que permiten justificar la acción y comprender el mundo social que los rodea.

Para acceder a las subjetividades a través de los estudios cualitativos se utilizan técnicas como: las entrevistas, los grupos focales, la observación, entre otras. Las preguntas que generalmente se pretenden despejar son: ¿cómo comprendemos la acción de los sujetos?, ¿qué sentido le dan a la acción que hacen? Por ello, elegimos el formato cualitativo.

El presente estudio cualitativo es descriptivo y exploratorio, sustentado en la construcción de los discursos narrativos y las percepciones de las adolescentes, en este caso, a través de los grupos focales. Los mismos que tienen como propósito construir representaciones sociales sobre un tópico o problema mediante una discusión abierta entre sus participantes.

\section{DESCRIPCIÓN METODOLOGIA}

\section{Objetivo General}

Interpretar los significados del riesgo sexual de las adolescentes de Lima que acuden a espacios de diversión dentro de un contexto donde hay alcohol y drogas.

\section{Objetivos específicos}

- Describir los significados de las drogas y el alcohol en las interacciones sociales en las adolescentes.

- Describir las interpretaciones sobre el significado de riesgo sexual en las ado- 
lescentes.

- Describir las interrelaciones sociales que se establecen en el contexto de diversión donde puede existir la presencia de drogas y/o alcohol.

\section{Población de estudio}

La población del estudio estuvo compuesta por adolescentes mujeres que asistan a las academias pre-universitarias del centro de Lima. El criterio de selección se basó en informantes clave.

\section{Muestra}

Se realizaron 8 grupos focales conformados por 8 adolescentes cada uno (64, muestra total); las edades estuvieron comprendidas entre los 15 y 18 años. Las entrevistadas se distribuyeron por estrato socioeconómico de la siguiente manera ${ }^{1}$.

\section{Cuadro 1}

Distribución de la muestra

\begin{tabular}{|c|c|c|c|}
\hline $\begin{array}{c}\text { Población } \\
\text { 64 }\end{array}$ & Distritos & $\begin{array}{c}\text { Sector } \\
\text { Socio econ. }\end{array}$ & Porcentaje \\
\hline 45 & $\begin{array}{c}\text { San Juan de Lurigancho, Villa María del Triunfo,Comas, } \\
\text { Callao, Puente Piedra, Chorrillos, Pachacamac, San Juan } \\
\text { de Miraflores, Vitarte y Santa Anita. }\end{array}$ & Bajo & $70 \%$ \\
13 & San Martín de Porras, San Luis, Rímac y Surquillo & Medio & $20 \%$ \\
4 & Lince y Jesús María. & Medio Alto & $6 \%$ \\
2 & La Molina. & Alto & $3 \%$ \\
\hline
\end{tabular}

El número tan elevado de entrevistadas (64), para una investigación de este tipo, tuvo por finalidad otorgar la mayor validez y confiabilidad posible a los resultados.

\section{Procedimiento de la realización de los grupos focales}

El centro de Lima, comprendido entre las avenidas Wilson, Paseo Colón, Alfonso Ugarte y Nicolás de Piérola, conforma un cuadrilátero imaginario donde se reúne la mayoría de las academias preuniversitarias del centro de Lima. Es por ello que se realizaron varios recorridos con el fin de determinar la zona donde se congregan mayor cantidad de adolescentes, ya sea a través de las mencionadas academias pre-universitarias, o de las discotecas de la zona.

Se hicieron coordinaciones con tres academias pre-universitarias que se localizan en la Av. Wilson con Paseo Colón. En todos los casos la recepción encontrada fue muy positiva, pero nos decidimos por la academia con mayor cantidad de alumnos y con mejor ubicación respecto de la esquina mencionada. Una vez seleccionada la academia, se formalizó un compromiso de confidencialidad tanto del centro de estudios como de las entrevistadas. Luego, se procedió a elaborar un cronograma de los grupos focales entre el mes de julio y agosto del año 2000. Es de señalar la gran colaboración que en todo momento mostró la academia pre-universitaria y del personal de la misma.

\footnotetext{
${ }^{1}$ Fuente: Planos estratificados de Lima Metropolitana (según estratos socio económicos) INEI/MOSAICO MARKETING PERU S.A.
} 
La selección de las adolescentes fue realizada a través del responsable de la programación de estudios de la academia pre-universitaria. Se le entregó al tutor un documento que contenía los criterios de selección de las participantes y éste se encargó de señalar las alumnas, la fecha, la hora y el lugar de reunión. La participación fue voluntaria.

Los grupos focales se realizaron en un espacio acondicionado en el segundo piso de la academia. Se contaba con dos moderadores de los grupos focales y dos observadores no participantes quienes, además, tomaban apuntes para facilitar la transcripción posterior. Se informó a las asistentes que la participación era voluntaria, anónima y confidencial. También se solicitó el consentimiento para proceder a la grabación en cintas de audio con una grabadora portátil; en ningún caso hubo objeciones.

Se elaboró una guía de discusión para la realización de los grupos focales. Esta se construyó sobre la base de los objetivos del estudio y se orientó a levantar datos sobre las siguientes categorías de análisis:

\section{Percepciones sobre los significados de las drogas y el alcohol}

Significados sobre los contextos de diversión donde hay consumo de alcohol y drogas.

Significados sobre la sexualidad, las prácticas sexuales, parejas sexuales y uso del condón.

\section{Procesamiento y análisis de los datos}

La información registrada en grabaciones de audio fue transcrita de la manera más fidedigna posible, tomando en cuenta los signos de puntuación, exclamación, los silencios. Esto se denomina: reducción de datos.

Luego se procedió a levantar un libro de códigos para codificar las entrevistas y los grupos focales, para ello se utilizó el paquete cualitativo Atlas Ti.

A partir de las entrevistas y grupos focales codificados se elaboraron documentos intermedios por cada grupo focal y entrevista. Estos documentos intermedios permitieron elaborar el informe final de investigación y complementar los resultados.

Planteamos una síntesis sobre los tres objetivos específicos de la investigación, con la finalidad de difundir sobre qué presupuestos teóricos nos hemos basado para la realización de la presente investigación.

\section{RESULTADOS ALCOHOL}

Investigar sobre la relación que existe entre el consumo de drogas y alcohol en el interior de sus relaciones sociales y sexuales fue una de los motivos que impulsó a realizar el presente estudio. La escasez de investigaciones que nos permitan conocer procesos como: el primer consumo de drogas o alcohol; los primeros episodios de embriaguez; las nuevas formas de relacionarse en parejas, son sólo alguno de los temas que desarrollamos en los cinco capítulos del estudio.

En lo concerniente al alcohol el primer consumo se realiza en la familia, inducido por un familiar generalmente de sexo masculino y se da alrededor de los 7 años. Es alrededor de los 15 años donde la adolescente inicia el consumo o abuso por propia voluntad. Los espacios sociales donde se producen estos episodios de consumo o embriaguez son: la culminación de la etapa escolar, los quinceañeros, los paseos y fiestas de promoción de secundaria. 


\section{Cuadro 2}

\section{Principales características del consumo de Alcohol}

\begin{tabular}{|l|l|l|l|l|c|}
\hline $\begin{array}{c}\text { Consumo } \\
\text { de alcohol } \\
\begin{array}{c}\text { Categoría de } \\
\text { consumo } \\
\text { y/o abuso }\end{array}\end{array}$ & $\begin{array}{c}\text { Espacio público } \\
\text { de padreso } \\
\text { adultos }\end{array}$ & Controles & Bebida & Presencia & Fdad \\
\hline Primer consumo & Familia & $\begin{array}{l}\text { Muy fuerte } \\
\text { por parte de } \\
\text { los padres }\end{array}$ & Cócteles, vinos & Presentes & $\begin{array}{c}\text { 5años en } \\
\text { en adelante }\end{array}$ \\
\hline Picada & Amigos(as) & Escaso & $\begin{array}{l}\text { Cerveza, Ron } \\
\text { Pisco }\end{array}$ & Ausentes & 15 \\
\hline $\begin{array}{l}\text { Embriaguez } \\
\text { moderada }\end{array}$ & Amigos(as) & Escaso & $\begin{array}{l}\text { Cerveza, Ron } \\
\text { Pisco }\end{array}$ & Ausentes & 15 \\
\hline $\begin{array}{l}\text { Embriaguezo } \\
\text { borrachera }\end{array}$ & Amigos(as) & Escaso & $\begin{array}{l}\text { Cerveza, Ron } \\
\text { Pisco }\end{array}$ & Ausentes & 15 \\
\hline
\end{tabular}

Los escasos niveles de autocontrol se demuestran en el hecho de que la chica bebe alcohol hasta situarse por igual en la categoría de PICADA, EMBRIAGUEZ MODERADA O EMBRIAGUEZ. Es claro que no hay un limite en la ingesta de alcohol ni de sus efectos en el organismo.

\section{Cuadro 3}

\section{Principales características del consumo de Alcohol}

\begin{tabular}{|c|c|c|}
\hline \begin{tabular}{c|} 
Concentración \\
en sangre
\end{tabular} & $\begin{array}{l}\text { Equivalencia en vasos } \\
\text { y/o botellas de cerveza }\end{array}$ & Efectos producidos \\
\hline $0.05 \%$ & $\begin{array}{l}\text { Equivale a la ingestión de } 3 \\
\text { tragos o } 9 \text { vasos de cerveza } \\
\text { en un lapso de } 2 \text { horas. }\end{array}$ & $\begin{array}{l}\text { Pérdida del proceso cortical: alteración de la capacidad de } \\
\text { juicio y pensamiento. Desinhibición. Se liberan las tensio- } \\
\text { nes y se produce una sensación de despreocupación, se da } \\
\text { menos importancia a la crítica de los que nos rodean. }\end{array}$ \\
\hline $0.10 \%$ & $\begin{array}{l}\text { Equivale a la ingestión de } \\
5 \text { tragos o } 15 \text { vasos de } \\
\text { cerveza en un lapso de } \\
2 \text { hrs. }\end{array}$ & $\begin{array}{l}\text { La actividad motora voluntaria es afectada: Se pierde } \\
\text { fluidez en los movimientos de las manos y los brazos, } \\
\text { así como también al hablar o caminar. Hay torpeza } \\
\text { motora, reacciones lentas. Emociones exageradas y en- } \\
\text { torpecimiento de la visión. }\end{array}$ \\
\hline $0.20 \%$ & $\begin{array}{l}\text { Equivale a la ingestión de } \\
10 \text { tragos o } 30 \text { vasos de cer- } \\
\text { veza en un lapso de } 2 \text { hrs. }\end{array}$ & $\begin{array}{l}\text { Hay un severo deterioro, se camina tambaleando. Sensa- } \\
\text { ción de tener que estar recostado, inhabilidad de estar de } \\
\text { pie o caminar sin ayuda. Habla en voz alta e } \\
\text { incoherentemente; se pierde la estabilidad emocional, co- } \\
\text { rajeo lágrimas pueden presentarse fácilmente. La persona } \\
\text { está ebria. El riesgo de accidente al conducir en este estado } \\
\text { aumenta } 100 \text { veces. }\end{array}$ \\
\hline $0.30 \%$ & $\begin{array}{l}\text { Equivale a la ingestión de } \\
15 \text { tragos o } 45 \text { vasos de cer- } \\
\text { veza en } 2 \text { hrs. }\end{array}$ & $\begin{array}{l}\text { Las áreas más profundas del cerebro son afectadas, lo } \\
\text { que altera las respuestas a los estímulos, la capacidad } \\
\text { de entendimiento es confusa. Decaimiento cercano a la } \\
\text { inconsciencia, no comprende lo que ve o lo que oye. }\end{array}$ \\
\hline $0.40 \%$ & $\begin{array}{l}\text { Equivale a la ingestión de } \\
20 \text { tragos o } 60 \text { vasos de } \\
\text { cerveza en } 2 \text { hrs. }\end{array}$ & $\begin{array}{l}\text { Dormido, dificultad de despertarse; incapacidad de ac- } \\
\text { tuar voluntariamente. Semejante al estado producido } \\
\text { cuando se está bajo los efectos de anestesia quirurgica. } \\
\text { Inconsciencia. Shock. }\end{array}$ \\
\hline $0.50 \%$ & $\begin{array}{l}\text { Equivale a la ingestión de } 24 \\
\text { tragos o } 72 \text { vasos de cerveza } \\
(9 \text { bos }) \text { en un lapso de } 2 \text { hrs. }\end{array}$ & $\begin{array}{l}\text { Coma: en los hospitales se trata de controlar los latidos } \\
\text { del corazón y la respiración. Alto riesgo de morir. }\end{array}$ \\
\hline
\end{tabular}


Con el fin de uniformizar criterios con relación al término "trago" y debido a la multiplicidad de bebidas alcohólicas, planteamos las siguientes equivalencias: 3 vasos de cerveza equivalen a 1 vaso colmado de vino y a 1 copa de whisky, pisco, ron o vodka (véase el cuadro precedente), más de este consumo nos coloca en riesgo de accidente al conducir un vehículo motorizado.

Con relación a las drogas ilegales, la marihuana se constituye en la droga ilegal de mayor accesibilidad a las adolescentes. Tanto en su disponibilidad como en su ofrecimiento están presentes los grupos de amigos, y aunque este hecho no se correlaciona con un consumo en las adolescentes, es claro que la marihuana es la droga ilegal con mayor permisividad social, incluso maestros y tutores hacen expresa defensa de su consumo frente a otras drogas como el tabaco.

La pasta básica de cocaína, no tiene una presencia relevante a lo largo del estudio, al parecer para la mujer resulta poco atractiva, y, por el contrario, hay en ella una actitud de rechazo comparándola con la actitud que tienen hacia la marihuana.

A pesar de ser una droga introducida recientemente en nuestro país, el estudio revela presencia no de consumo directo pero si en el entorno de esta droga. Este consumo esta difundiéndose cada vez más a sectores populares, rompiéndose así el mito del éxtasis como droga "pituca".

\section{RESULTADOS SEXUALIDAD}

La sexualidad adolescente, si bien si ha recibido más atención a través de estudios e investigaciones de diferentes instituciones, nuestro interés particular estuvo centrado en encontrar las nuevas formas de relaciones de pareja en espacios de diversión, así como identificar e interpretar los significados que la sexualidad tiene en las adolescentes.

Así, el varón es percibido por las adolescentes como un ser sometido y conducido a sus impulsos sexuales de origen biológico. Las mujeres en cambio tendrían la capacidad de controlar estos impulsos. Por otro lado, la virginidad tiene una doble interpretación para las adolescentes, por un lado se encuentran las mujeres con una actitud menos tradicional que manifiestan que más importante que la virginidad son los aspectos relacionados a la relación de pareja. Por el otro, están las adolescentes más tradicionales que manifiestan que la virginidad hay que cuidarla y debe entregarse al hombre que se ama, pero al mismo tiempo afirman no saber que podría pasar en el futuro sobre su sexualidad.

\section{Cuadro 4 \\ Significado de las relaciones sexuales}

\begin{tabular}{|l|c|c|c|c|}
\hline $\begin{array}{l}\text { Relaciones } \\
\text { sexuales }\end{array}$ & $\begin{array}{c}\text { Tipo de chicas } \\
\text { de "los otros" }\end{array}$ & $\begin{array}{c}\text { Tipode vínculo } \\
\text { sentimental }\end{array}$ & Actitud & Significado \\
\hline Por placer & $\begin{array}{c}\text { «jugadora» } \\
\text { «vacilonera» }\end{array}$ & $\begin{array}{c}\text { «vacilones» } \\
\text { (vínculo débil) }\end{array}$ & Desaprobación & «lujuria» \\
\hline Por amor & $\begin{array}{c}\text { «tranquila» } \\
\text { «de su casa» }\end{array}$ & $\begin{array}{c}\text { «enamorados» } \\
\text { (vínculo fuerte) }\end{array}$ & Comprensión & «entrega de pureza» \\
\hline
\end{tabular}


En este sentido, el mayor temor que ocasiona mantener relaciones sexuales no son las enfermedades de transmisión sexual, sino el embarazo, ya que éste último no es posible ocultarlo por mucho tiempo, y el temor reside, justamente en la reacción de la familia ante ese hecho.

La percepción de la mujer como objeto sexual continúa siendo un problema social, ya que la mayoría de las entrevistadas acepta haber sido objeto de agresiones sexuales, en diferentes espacios públicos como son la calle, los vehículos de transporte, e incluso al interior de su familia.

Con relación a las formas de relación de pareja que se establecen en los espacios de diversión, las hemos clasificado en tres tipos de relaciones: los agarres, los vacilones y la relación de enamorados.

\section{Cuadro 5}

\section{Dimensión de las relaciones y tipos de relaciones de pareja}

\begin{tabular}{|l|l|l|l|l|l|l|}
\hline $\begin{array}{l}\text { Dimensión de } \\
\text { las relaciones } \\
\text { Tipode relación }\end{array}$ & $\begin{array}{l}\text { Espacio } \\
\text { público }\end{array}$ & Obligatoried & Afectividad & Erotismo & $\begin{array}{c}\text { Contacto } \\
\text { físico }\end{array}$ & $\begin{array}{l}\text { Tiempo de } \\
\text { duración }\end{array}$ \\
\hline Agarre & $\begin{array}{l}\text { En los espacios } \\
\text { de diversión }\end{array}$ & Débil & Débil & Fuerte & Débil & $\begin{array}{l}\text { Uno o va- } \\
\text { rios momen } \\
\text { tos de la } \\
\text { reunión }\end{array}$ \\
\hline Vacilón & $\begin{array}{l}\text { Va más allá de los } \\
\text { espacios de diversion } \\
\text { pero sin entrar a } \\
\text { espacios públicos o } \\
\text { privados de la chica } \\
\text { odel chice }\end{array}$ & Débil & Débil & Fuerte & Fuerte & $\begin{array}{l}\text { Una noche } \\
\text { a varias } \\
\text { semanas }\end{array}$ \\
\hline $\begin{array}{l}\text { Enamorados } \\
\text { Comparten sus } \\
\text { espacios públicos y } \\
\text { privados }\end{array}$ & Fuerte & Fuerte & $\begin{array}{l}\text { Más o } \\
\text { menos } \\
\text { fuerte }\end{array}$ & Fuerte & $\begin{array}{l}\text { De meses } \\
\text { a años }\end{array}$ \\
\hline
\end{tabular}

La jugadora, la sangrona, la fácil y la vacilonera son descritas como formas de "ser mujer", alternativas al rol tradicional. La sanción que hacen de ellas dependerá si cada una de estas formas están acompañadas o no, de manoseos, juegos eróticos o coito.

\section{Cuadro 6}

Tipos de chicas según su interacción sexual

\begin{tabular}{|l|c|c|}
\hline Tipo de relación & $\begin{array}{c}\text { Relaciones sexuales } \\
\text { penetrativas }\end{array}$ & $\begin{array}{c}\text { Relaciones sexuales } \\
\text { No penetrativas }\end{array}$ \\
\hline Agarre & Rucas & $\begin{array}{c}\text { Vaciloneras } \\
\text { Sangronas }\end{array}$ \\
\hline Vacilón & Rucas & $\begin{array}{c}\text { Tramposa, Fácil } \\
\text { Sangronas }\end{array}$ \\
\hline
\end{tabular}


La discoteca es un espacio que reúne características apreciadas por las adolescentes como: divertirse sin remordimiento, vivir el momento, hacerla dentro de horas de estudio (evitando sanciones familiares o de los adultos en general). Y por otro, es percibida como un espacio de cierto riesgo manejable donde la posibilidad de ser pepeadas y violadas esta presente, pero con la adopción de medidas de seguridad individuales y grupales son superables.

\section{Cuadro 7}

\section{Características de la discoteca como espacio de diversión}

\begin{tabular}{|l|l|}
\hline *El espacio de la diversión sin culpa & - Las adolescentesperciben como un espacio \\
* Del sistema de amigos & de peligro pues tienen el temor a ser "pepeadas* \\
* La vivencia hedonista & -Se percibe como un espacio de la \\
* La vivencia y racionalidad pragmática & desconfianza ante los amigos, por el temor \\
* Del presente constante & a ser violadas. \\
* La noche artificial & -Se percibe como el espacio del eno-tiempo \\
* Lejos de las normas sociales de los adultos & librem. \\
\hline
\end{tabular}

\section{CONCLUSIONES}

\section{Drogas y Alcohol}

1. El inicio del consumo del alcohol se realiza en el ámbito familiar, ésta propicia y/o permite sin mayor control dicho consumo. Esto podría explicar el porqué es que el inicio del consumo de alcohol se esta reduciendo progresivamente. Un hecho a investigar en la familia es que este primer consumo es inducido por una figura masculina.

2. La primera embriaguez no es planificada por las adolescentes. Sucede de forma inesperada lo cual confirmaría el riesgo potencial que el consumo de alcohol representa.

3. No existe autocontrol frente al consumo de esta sustancia. Los resultados obtenidos a lo largo de la investigación confirman que existe una relación estrecha entre la permisividad social frente al consumo de alcohol y el escaso autocontrol observado en las adolescentes.

4. Si bien la embriaguez se produce en la etapa escolar / adolescente, es alrededor de los 15 años que se da con mayor intensidad. Los espacios señalados por las entrevistadas para este episodio son: el término del colegio (reuniones programadas), la fiesta de promoción, los quinceañeros y lo paseos de promoción.

5. Con relación a las drogas ilegales, el estudio confirma que los amigos se constituyen en el grupo social donde se da por primera vez el ofrecimiento y/o la disponibilidad para el consumo de la marihuana. Es preocupante la actitud de permisividad hacia la marihuana que existe en las adolescentes, si bien esta actitud no se traduce en un consumo directo, la percepción de la marihuana es sinónimo de droga suave, blanda o inofensiva.

6. Otro hallazgo sumamente interesante es el que nos indica que modelos decisivos para toda adolescente como tutores o maestros manifiesten abiertamente una posición favorable hacia la marihuana calificando al tabaco como más peligroso o dañino que la

En relación a la PBC sigue siendo la droga menos cercana a las adolecentes, esto debido a que no resulta tan atractiva en relación con otras drogas, ya que su consumo está relacionado con actitudes negativas y de rechazo más definidas.

La presencia del éxtasis se está extendiendo cada vez más hacia sectores $\mathrm{C}$ y $\mathrm{D}$, 
apartándose rápidamente de los sectores A y $\mathrm{B}$ donde inicialmente apareció. Las discotecas populares y la aparente adulteración de las pastillas de éxtasis son una combinación que está potenciando su abuso notoriamente.

\section{Actitudes hacia la sexualidad}

1. Las adolescentes perciben que el deseo sexual masculino es muy intenso y es el motivo de que los varones realicen una serie de conductas reprobables. Las mujeres no tendrían un deseo sexual de esa misma intensidad o en todo caso "lo sabrían controlar".

2. Coexisten dos discursos sobre la virginidad: uno, el más predominante, la considera muy importante y que debería cuidarse, y otro que le resta importancia y que le otorga más relevancia a las cualidades personales en una relación. Sin embargo, quienes coincidían con la primera posición manifestaban no saber que podría pasar con su virginidad en el futuro. Trasladando así su decisión y responsabilidad al "destino o futuro incierto".

3. La consecuencia de tener relaciones sexuales más temida por estas adolescentes es el embarazo. Esto se debe a que el embarazo tiene efectos más inmediatos y notorios. El mayor temor familia al percatarse del embarazo. Mientras que las enfermedades pueden mantenerse más o menos ocultas y el VIH tarda años en manifestarse, así las familias desconocerían el hecho.

4. El uso planificado de métodos anticonceptivos es asociado con un comportamiento sexual activo y asumido como liberal; de allí que pueda haber cierta resistencia a utilizarlos (en varones y mujeres). Esto se ve claramente en el caso del condón. La aparición e intento de uso del preservativo dificultará que el acto sexual se realice. (la mujer no se "deja llevar por el deseo" sino que racionalmente aceptaría tener relaciones, lo cual muchas evitan). De allí la actitud poco favorable hacia su uso. (en el varón se da el mismo razonamiento pero a la inversa, ... "si el condón evita que se deje llevar mejor no lo uso ... ") Es decir, el problema del poco uso del condón no es sólo de información o acceso, sino de actitud, fundamentalmente.

5. El espacio de discusión e información sobre temas de sexualidad más cercano para estas adolescentes son sus grupos de amigos contemporáneos. Este se caracteriza por el clima lúdico de la comunicación y el reforzamiento de esquemas ya establecidos sobre el tema.

6. La mayoría de las adolescentes ha sufrido algún tipo de agresión sexual en lugares como: su casa, en las calles, unidades de transporte público y otros lugares. Esto les hace sentir vulnerables frente a los hombres, lo cual refuerza su concepciones de varón (como sujeto de deseo y poder) y de mujer(como objeto de deseo).

\section{Formas de relaciones de pareja en los espacios de diversión}

Existen tres tipos de relaciones de pareja definidos por las adolescentes: agarres, vacilones y enamorados.

1. La relación que denominan «agarres», esta basado en vínculos débiles, anónimos y cierta atracción erótico/físico. El «agarre no va más allá de los besos cortos que se dan generalmente en el espacio de la discoteca.

En las relaciones de vacilón el espacio público es más amplio pero no entran al mundo del barrio, los estudios, la familia o el mundo privado. No llega a constituirse en una pareja sólida con derechos, las manifestaciones de afectividad es mínima. 
Establecen vínculos fuertes a través de la atracción erótica / física que es la base de esta forma de relación al igual que el tiempo de duración. Las relaciones de enamorados esta basado en el modelo del amor romántico y el vínculo que cobra mayor fuerza es el afectivo.

2. Existen diversas formas alternativas al rol tradicional de la mujer: jugadora, sangrona, fácil, vacilonera, que no son sancionados moralmente por la mayoría de las adolescentes. La sanción moral surge cuando en este tipo de interacción se dan relaciones sexuales penetrativas o demasiado contacto físico, como son los manoseas, juegos eróticos o besos apasionados.

3. La discoteca es el espacio de la diversión sin culpa, de la vivencia hedonista y pragmática, del presente constante, de la noche artificial, del baile lúdico, el «no-tiempo libre» (ya que van a estos espacios en horas de estudio), lejos de las normas sociales de los adultos.

4. La discoteca es percibida como un espacio de riesgo por parte de los padres, pero no por parte de las adolescentes. Si bien hay desconfianza por las otras personas que asisten al local e incluso por sus propios amigos, y hay temor a ser «pepeadas» y violadas. Existe la actitud que estos peligros se encuentran en cualquier espacio de diversión, incluida la discoteca y que por tanto se debe tomar medidas de seguridad y protección al asistir a la discoteca. La idea de dejar de ir a una discoteca no es siquiera contemplada.

\section{BIBLIOGRAFÍA}

Agrias, R. Y Agrambugrú, C. Uno empieza a alucinar.... Percepciones de los jóvenes sobre sexualidad, embarazo y acceso a los servicios de salud: Lima, Cuzco e Iquitos. Lima: Redess Jóvenes / Fundación Summit. 1999

Bardales, 1. Y Chirinos, K. Estudio de las necesidades y expectativas de un grupo de madres adolescentes de 15 a 19 años residentes en el distrito de El Agustino. Tesis para optar al título de Licenciado en Trabajo Social. Lima: PUCP. 1998.

Cáceres, C .. Nuevos retos: Investigaciones recientes sobre salud sexual / reproductiva de los jóvenes en el Perú. Lima: Redess Jóvenes. Corporación Colombiana para la Prevención del Alcoholismo y la Fármaco dependencia (SURGIR). 1999.

Callirgos, Juan Carlos. "Sobre héroes y batallas. Los caminos de la identidad masculina. Escuela para el desarrollo. Ira edición. Lima, diciembre de 1996.

Clemente, Raúl Editores c.A.,. Vol. 3 t.. Universidad de Carabobo. Memorias: I Congreso Internacional de «Educación Superior en el Problema de las Drogas. 3 t. Carabobo, 1992.

Cortázar, Juan Carlos. "La juventud como fenómeno social". Pistas teóricas para comprender el período juvenil en el Perú. Centro de Investigaciones Sociales, Económicas, Políticas y Antropológicas (CISEPA). 1997

Chau-Pérez-Aranibar, Cecilia Beatriz. Consumo de bebidas alcohólicas en estudiantes universitarios: motivaciones y estilos de afrontamiento. Tesis presentada en la Pontificia Universidad Católica del Perú para optar al Grado de Magister en Psicología. 1998.

Dusek E. Dorothy, Girdano, A. Daniel Drogas un estudio basado en hechos. México: SITESA (4ta. Edición). 1990. 
DROGADICCIÓN: Se eleva el consumo entre los adolescentes. Santiago de Chile, julio de 2000. www.saludpublica.com/ampll/ ampI08/juI58.htm.

Edwards, G. et al.. Alcohol y salud pública. Barcelona: Prous Science. 1997.

Figueroa, Juan Guillermo. "Algunas Propuestas analíticas para interpretar la presencia de los varones en los procesos de salud reproductiva". En: VALDES.

García Rodriguez, José, A.; Ruiz Fernández, Javier Comp. Tratado sobre prevención de las drogodependencias. Madrid, Fundación de ayuda contra la drogadicción, 1993. 398p.

González Menéndez, Ricardo, Revista Cubana de medicina general integral. Julio septiembre 1995. Cómo librarse de los hábitos tóxicos. http://www.sld.cu/revistas/ mgi/mgi06395.htm.

Instituto sobre Alcoholismo y Fármaco dependencia. Adolescencia y Consumo de Drogas en Costa Rica. Universidad Nacional; Instituto del Niño.121 p.tab .. Costa Rica. 1995.

Jiménez, Oscar. Más allá de la intimidad: cinco estudios en sexualidad, salud sexual y reproductiva. Lima. PUCP. 1996.

Lagarde, Marcela. Identidad de Género. Mimeo. Curso ofrecido en el Centro Juvenil "Olof Palme. Managua - Nicaragua del 25 al 30 de abril de 1992. OCSD-OIT-OPS-AOS, 1992.

Laufer, M .. Body image, sexuality and psychotic core. Londres: Institute of Psychoanalysis. 1991.

López, Hartmann, Rodolfo. Marcadores biológicos del alcoholismo. Fuente:

Psicoactiva, v. Lima 14. 67-80, ene - dic. 1996.

Lutte, G. Liberar la adolescencia. La psicología de los jóvenes de hoy. Barcelona:

Herder. 1991.

Margulis, Mario y Urresti, Marcelo. "La Juventud es más que una palabra". En: La Juventud es más que una palabra. Ensayos sobre cultura y juventud. Editorial Biblios. Bs. As. Argentina, 1996

Ministerio de Salud. División de Programas de Salud. Manual: orientaciones para un programa comunal de prevención del uso indebido de drogas y alcohol. Santiago de Chile, Ministerio de Salud, OCL 1995.72p.

Mendoza, Sagrega y Batista: Conductas de los escolares españoles relacionadas con la salud, 1986-1990. Madrid, 1994.

Perales, A. et al. Conductas de riesgo en adolescentes de Lima. en: Monografías de Investigación Nro. 6, Instituto de Salud Mental Honorio Delgado - Hideyo Noguchi. 1997.

Psicoactiva, v.:5-23, dic. tabModificación de los efectos del alcohol por la manipulación de la información acerca del contenido de la bebida en un grupo de estudiantes universitarios. Lima $15,1997$.

Pons Diez y Gil La cruz. Universidad de Zaragoza y Valencia. España. 2000. La cultura del alcohol. El consumo de bebidas alcohólicas pone en grave riesgo a los adolescentes. http://www.webislam.com/98/2610 02.htm

Quintana, A. y Vásquez, R. (1997). La construcción social de la sexualidad adolescente: Género y salud sexual. Lima: Instituto de educación y salud.

Raguz, María. Construcciones sociales y psicológicas de mujer, hombre, feminidad, masculinidad y género en diversos grupos poblaciones. Lima: Pontificia Universidad Católica del Perú. 1995.

Sebastiani, Angela. et al. Qué hacen, qué piensan, qué sienten los y las adolescentes de Lima 
respecto a la salud sexual y re productiva. Lima: INPPARES. 1997.

Surgir; Edex Kolektiboa. Cómo orientar a jóvenes que usan licor y otras drogas. Medellín; editorial Cooperación Española, ene. 45p.ilus: Colombia. 1997.

Teresa y Olavarria, José (Eds). "Masculinidades y equidad de género en América Latina". FLACSO - UNFPA. Santiago de Chile, 1998

U.N.M.S.M. Facultad de Farmacia y Bioquímica. Centro de Información, Control Toxicológico y Apoyo a la Gestión Ambiental. CICOTOX. Determinación toxicológica de drogas y metabolitos de cocaína y marihuana en escolares de educación secundaria. Lima - Perú 2000.

Vega-Centeno, I. Amor y sexualidad en tiempos del SIDA. Lima: MINSA. 1994. 